\title{
The Relationship Between Crackle Characteristics and Airway Morphology in COPD
}

\author{
Surussawadi Bennett PhD, Anne Bruton PhD, Anna Barney PhD, \\ Tom Havelock BMBS MRCP, and Michael Bennett PhD
}

\begin{abstract}
BACKGROUND: Crackles in COPD are believed to be generated by the re-opening of collapsed airways, which result from chronic inflammation, secretions, and loss of cartilaginous support through inflammation. However, it is unclear whether crackle characteristics can be used to identify COPD. This is the first study to examine the relationship between specific added lung sounds (crackles) and measurements of conductive airways and emphysema score obtained from highresolution computed tomography (HRCT) in vivo in humans. A predictive relationship might permit the use of lung sounds as a biomarker for COPD. METHODS: A convenience sample of 26 subjects was recruited into the study and consisted of 9 healthy non-smokers, 9 healthy smokers, and 8 subjects with mild or moderate COPD. Lung sound data were recorded using a digital stethoscope connected to a laptop computer. Airway diameter, emphysema score, and percentage of wall area were measured from HRCT scans. RESULTS: The analysis showed that there were no statistically significant differences in crackle characteristics (the number of crackles per breathing cycle and crackle 2 -cycle duration) between the 3 subject groups. Both crackle 2-cycle duration and crackle number showed some significant correlation with airway parameters at some branch generations, but due to the large number of correlations performed, these were consistent with chance findings. CONCLUSIONS: Although there were some significant correlations between crackle characteristics and measurements of the conductive airways and emphysema score, the possibility that these correlations have occurred by chance cannot be ruled out. Therefore, this study provides no conclusive evidence that crackle characteristics are related to HRCT variables in COPD. Key words: breathing sounds; crackles; lung sounds; COPD; cross-sectional anatomy; tomography, x-ray computed; imaging, 3-dimensional background. [Respir Care 2015;60(3):412-421. (C) 2015 Daedalus Enterprises]
\end{abstract}

\section{Introduction}

Use of standard auscultation to listen to lung sounds is an accepted component of routine clinical assessment, but

Dr S Bennett is affiliated with the Improvement of Physical Performance and Quality of Life Research Group, Division of Physical Therapy, Faculty of Associated Medical Sciences, Khon Kaen University, Khon Kaen, Thailand; Dr Bruton is affiliated with the Faculty of Health Sciences, University of Southampton, Southampton, United Kingdom; Dr Barney is affiliated with the Institute of Sound and Vibration Research, University of Southampton, Southampton; Mr Havelock is affiliated with the Faculty of Medicine, University of Southampton, Southampton; Dr Bruton, Mr Havelock, and Dr MJ Bennett are affiliated with the National Institute for Health Research, Southampton Respiratory Biomedical Research Unit, Southampton Centre for Biomedical Research, University it is a subjective process. Recent technological advances mean that lung sounds and their characteristics can now be recorded and objectively quantified, providing useful information on lung health. Crackles have been reported to

Hospital Southampton NHS Foundation Trust, Southampton, United Kingdom.

Supplementary material related to this paper is available at http:// www.rcjournal.com.

The clinical portion of this study was funded by the Southampton National Institute for Health Research Respiratory Biomedical Research Unit. Dr S Bennett received financial support from the Royal Thai Government (Ministry of Sciences) and Khon Kaen University. The other authors have disclosed no conflicts of interest. 


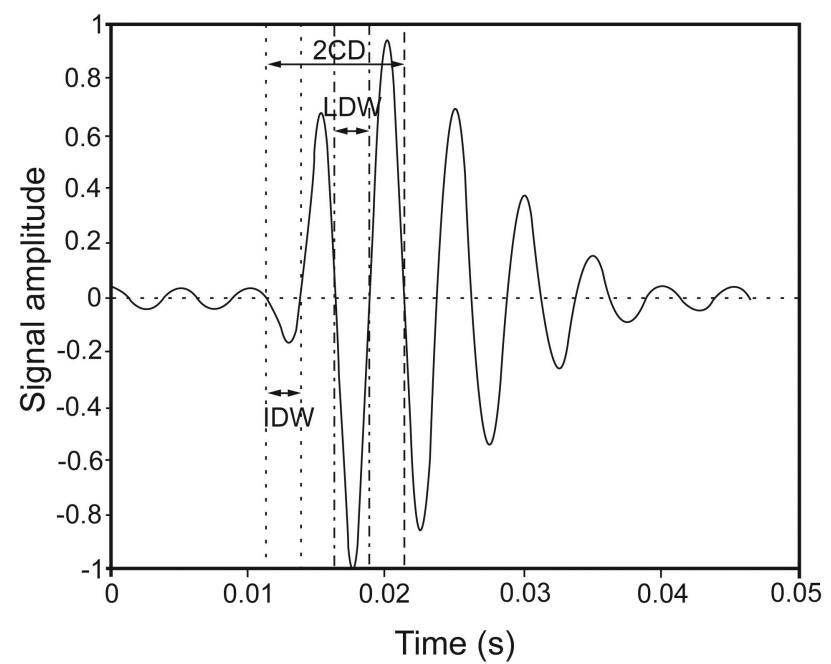

Fig. 1. Illustration of the crackle characteristics that were measured from the crackle sounds. $2 \mathrm{CD}=2$-cycle duration; IDW $=$ initial displacement width; LDW = largest deflection width.

be the most frequent of adventious lung sounds that are indicative of pulmonary diseases..$^{1-3}$

The mechanism to generate crackles is believed to be associated with the sudden reopening of closed airways, which result from secretion obstruction, bronchoconstriction, chronic inflammation, and loss of airway cartilaginous support. ${ }^{3,4}$ Crackles can be described in terms of duration (such as the crackle 2-cycle duration [2CD]; see Fig. 1), which is determined as the time from the beginning of a crackle to the point where the crackle has completed 2 cycles $^{5}$ and quantity (such as the number of crackles per breathing cycle $[\mathrm{NCpB}])^{1,3,6}$ Crackle duration is related to airway size, with smaller airways thought to give rise to crackles of shorter duration. ${ }^{3}$ The number of closed airways that reopen directly affects the crackle number, as each reopening event causes one or more crackles. The pressure required to cause sudden reopening of closed airways has been shown to be related to the airway diameter; 7,8 therefore, it can be hypothesized that the characteristics of crackles as described above may be related to airway geometry. COPD is a chronic inflammatory disease of the lungs that results in progressive narrowing of the airways, ${ }^{9}$ and it was therefore hypothesized that the charac-

Correspondence: Anne Bruton $\mathrm{PhD}$, National Institute for Health Research, Southampton Respiratory Biomedical Research Unit, Southampton Centre for Biomedical Research, Mailpoint 218, D Level, West Wing, University Hospital Southampton NHS Foundation Trust, Tremona Road, Southampton SO16 6YD, United Kingdom. E-mail: ab7@soton.ac.uk.

DOI: $10.4187 /$ respcare. 03543

\section{QUICK LOOK}

\section{Current knowledge}

The adventitious breath sounds known as crackles are believed to be associated with the sudden reopening of closed airways resulting from secretion obstruction, bronchoconstriction, chronic inflammation, and loss of airway cartilaginous support. Crackles are associated with COPD, atelectasis, and pulmonary edema.

\section{What this paper contributes to our knowledge}

There was no significant relationship between crackle characteristics quantified using computer-aided lung sound analysis and emphysema score measured by highresolution computed tomography. The presence and extent of crackles does not correlate with emphysema severity.

teristics of crackles would be altered in the presence of COPD.

Quantitative analysis of high-resolution computed tomography (HRCT) of the lungs has been demonstrated to be a useful method of characterizing and measuring the anatomy and geometry of the bronchial tree. Although the technique is limited by resolution in its assessment of the very small airways, it is reliable down to the sixth airway generation. ${ }^{10}$ Increased wall thickening in COPD is associated with worsening air flow limitation and increased inflammation ${ }^{11}$ and, as measured by quantitative computed tomography analysis, predicts increased symptoms and worse health outcomes. ${ }^{12}$ Thickening of the walls of the central airways correlates weakly with spirometric measures of airways obstruction, ${ }^{13}$ but the strength of the association increases as the geometric measurements are taken from more distal airways at the fifth and sixth generations. ${ }^{14}$

Airway wall thickness decreases with increasing airway generation due to the normal anatomy of the airways. ${ }^{11}$ Percentage wall area has been introduced as a way of normalizing the wall thickness measurement for different airway generations. It is calculated by expressing the total airway wall area as a percentage of the whole area enclosed by the external perimeter of the airway wall (airway wall area plus luminal area). The normalization is not perfect, however, as percentage wall area nevertheless has the tendency to increase with airway generation. ${ }^{15}$

The chronic inflammation associated with COPD also leads to destruction of the alveoli walls, causing the enlargement of the air spaces within the lung that is the defining feature of emphysema. Emphysema can be detected by HRCT as areas of low attenuation. ${ }^{16}$ The extent of emphysema in the lung can be quantitatively assessed 
from the HRCT by counting the number of voxels with an attenuation value less than a specified threshold. The most commonly used threshold is -950 Hounsfield units ${ }^{17}$ and the ratio of the number of voxels below this threshold (often referred to as low attenuation areas) to the total number of voxels in the lung (or region), expressed as a percentage is defined as the emphysema score.

Computer-aided lung sound analysis (CALSA) has been reported to provide good to excellent intra-subject reliability of measurements of crackle characteristics such as duration and number in a clinical population. ${ }^{6}$ CALSA is an inexpensive, noninvasive process with potential to be an objective diagnostic and monitoring tool for clinical practice. ${ }^{6,18}$ The aim of this study was therefore to explore the relationship between crackles (analyzed using CALSA) and airway geometry and lung parenchyma measurements (quantified by HRCT) to test whether crackles can be used as an indicator of COPD.

\section{Methods}

\section{Subjects}

A convenience sample of 26 subjects was recruited and characterized into disease groups relating to smoking habits and level of airway obstruction. Convenience sampling is a non-probability sampling technique where participants are recruited on the basis of their accessibility or proximity to the researcher. In this case, participants were recruited on the basis of being involved in an imaging study being conducted within University Hospital Southampton National Health Service Foundation Trust. Nine healthy nonsmokers, 9 healthy smokers without airway obstruction, and 8 subjects with mild or moderate COPD were in the sample. Healthy non-smokers were defined as life-long non-smokers, with no medical history suggestive of airway disease, normal spirometry, non-atopic, and $\mathrm{PC}_{20}$ (histamine) $>8 \mathrm{mg} / \mathrm{mL}$. Healthy smokers were defined using the same criteria as the healthy non-smokers, except they were current smokers. COPD subjects were defined as current or ex-smokers, with physiological evidence of mild or moderate obstructive airway disease $\left(\mathrm{FEV}_{1} / \mathrm{FVC}\right.$ ratio of $<70 \%$ and $\mathrm{FEV}_{1} \geq 50 \%$, predicted), incomplete disease reversibility $(<10 \%$ improvement with $400 \mu \mathrm{g}$ of inhaled salbutamol), symptoms of breathlessness, cough or sputum production, no evidence of exaggerated diurnal variation, atopy, or asthma.

\section{Ethics and Research Governance}

Written informed consent was given and signed by all subjects. The study was approved by the Southampton and South West Hampshire local research ethics committee
(LREC No. 09/H0502/91) and Southampton University Hospital Trust Research and Development department.

\section{Lung Sound Recording and Analyzing}

Single-channel sound recordings were made with a handheld digital stethoscope (ds32a, ThinkLabs, Centennial, Colorado). Lung sounds were recorded on the same day as HRCT imaging. The recordings were conducted as described in detail elsewhere ${ }^{6,18}$ using 6 anatomical regions of the chest wall: anterior right (AR) and anterior left (AL), lateral right (LR) and lateral left (LL), and posterior right (PR) and posterior left (PL) (see Fig. 2). A single microphone was used and moved sequentially to each anatomical region. The recording time lasted $25 \mathrm{~s}$ at each region, with subjects in a supine position (as for the HRCT procedure) without air flow control. Recording without air flow control has been reported to give high sensitivity and specificity in detecting crackles. ${ }^{6}$ Subjects were asked to breathe slightly more deeply than normal. The output from the stethoscope microphone was connected via an integral amplifier to the sound card of a laptop with customized software, suitable for data acquisition and analysis, written in Matlab 7.1 (Mathworks, Natick, Massachusetts). All lung sound files from the 6 areas of chest wall were processed using a customized algorithm written in Matlab, used in previous research. ${ }^{6,18}$ Crackle characteristics, ie, crackle $2 \mathrm{CD}$ and $\mathrm{NCpB}$, were exported into an Excel (Microsoft, Redmond, Washington) file for analysis.

\section{HRCT Measurement}

The HRCT scan was performed on a Sensation 64 computed tomographic scanner (Siemens Medical Solutions, Erlangen, Germany) with detector aperture $0.6 \mathrm{~mm}$, pitch 1 , effective $\mathrm{mAs}$ of 90 , and a tube voltage of $120 \mathrm{kV}$. The images were reconstructed using the Siemens b35f filter, and the effective dose for this procedure was approximately $3.8 \mathrm{mSv}$ to get the best visualization of the airway tree. ${ }^{19}$ Images were captured with subjects in suspended full inspiration and on full expiration. The combined radiation dose of the inspiratory and expiratory scans was approximately $7 \mathrm{mSv}$.

The analysis of the HRCT scans was performed using Pulmonary Workstation 2 (PW2) software (Vida Diagnostics, Coralville, Iowa), which was used to segment the airway, to give measurements of airway diameter and percentage wall area and measurement of emphysema score. Measurements were made for each branch of the airways from generation 2 to 5 at right upper, middle, and lower lobes, and from generation 2 to 4 at left upper and lower lobes because of the limitation of HRCT scan (see Figs. 3 and 4). 


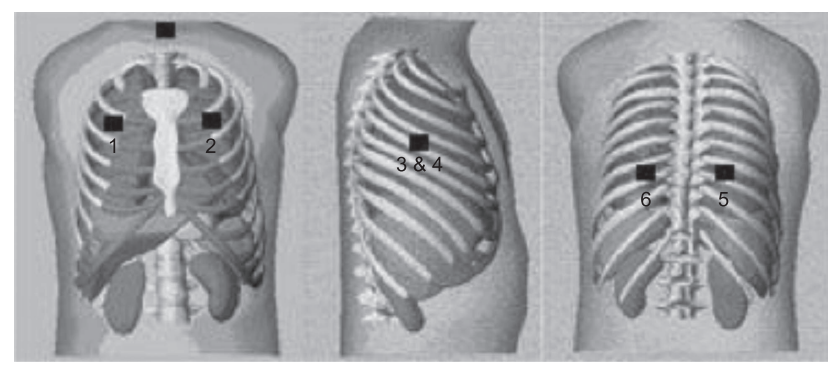

Fig. 2. Site of the 6 auscultation locations of the chest: 1: anterior right; 2: anterior left; 3: lateral right; 4: lateral left; 5: posterior right; 6: posterior left. (Adapted from Reference 6, with permission.)

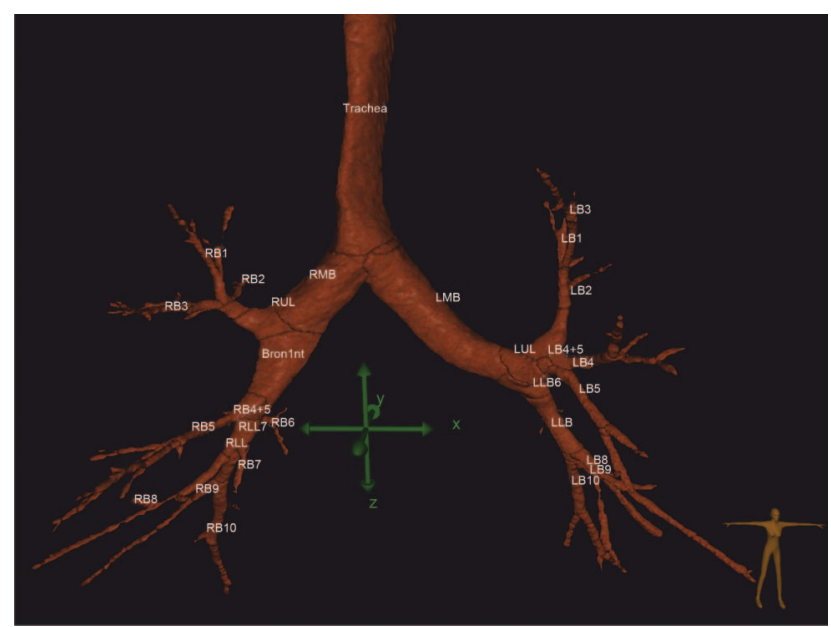

Fig. 3. Illustration of the tracheobronchial tree segmented from the HRCT data, showing automatic airway labeling by Pulmonary Workstation 2 (Vida Diagnostics). RMB = right main bronchus, LMB = left main bronchus, RUL = right upper lobe, $\mathrm{LUL}=$ left upper lobe, LLB = left lower bronchus, RB1-RB10 = right segmental bronchi, LB1-LB10 = left segmental bronchi.

The parameter used to describe airway wall thickening, obtained from the HRCT images is the percentage wall area (\%WA). The percentage wall area is defined as:

$$
\% \mathrm{WA}=100\left(\left[\mathrm{~A}_{\mathrm{ext}}-\mathrm{A}_{\mathrm{int}}\right] / \mathrm{A}_{\mathrm{ext}}\right)
$$

where $\mathrm{A}_{\text {ext }}$ and $\mathrm{A}_{\text {int }}$ are defined in Figure 5. The percentage wall area and airway diameter measurements were made automatically by the Pulmonary Workstation 2 software, which made measurements from more than one location along each airway branch and provided the average value.

The emphysema score, expressed as the percentage of voxels in each region that had a threshold less than -950 Hounsfield units, was computed for each lobe. The Pulmonary Workstation II software automatically segmented the lungs and attempted to automatically detect the fissures of the lungs and thus segment the lobes. In the ma-

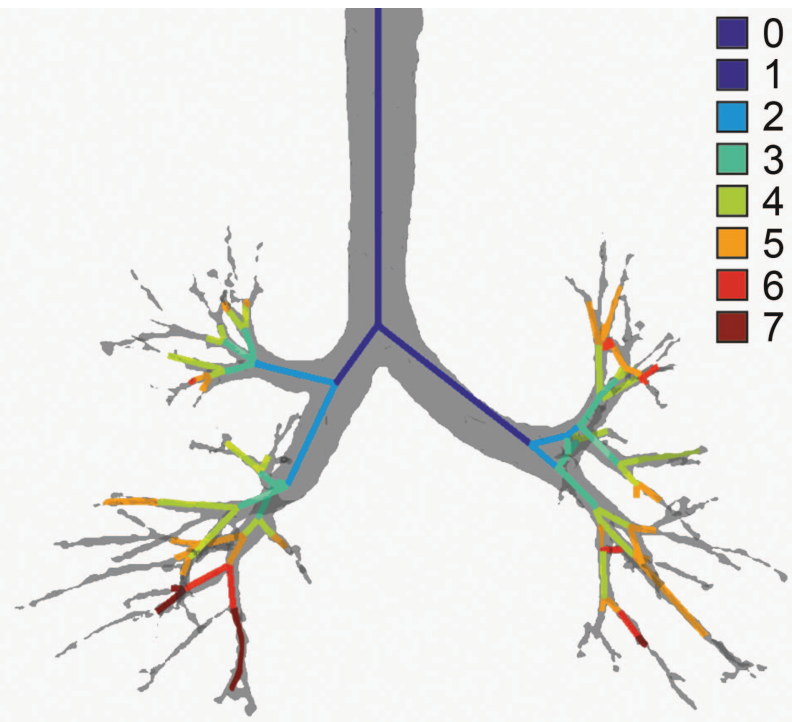

Fig. 4. Illustration of the different generations of the airway tree produced by Pulmonary Workstation 2 (Vida Diagnostics). $0=$ trachea, $1=$ left/right main bronchus, $2=$ generation $2,3=$ generation $3,4=$ generation $4,5=$ generation $5,6=$ generation 6 , $7=$ generation 7 .

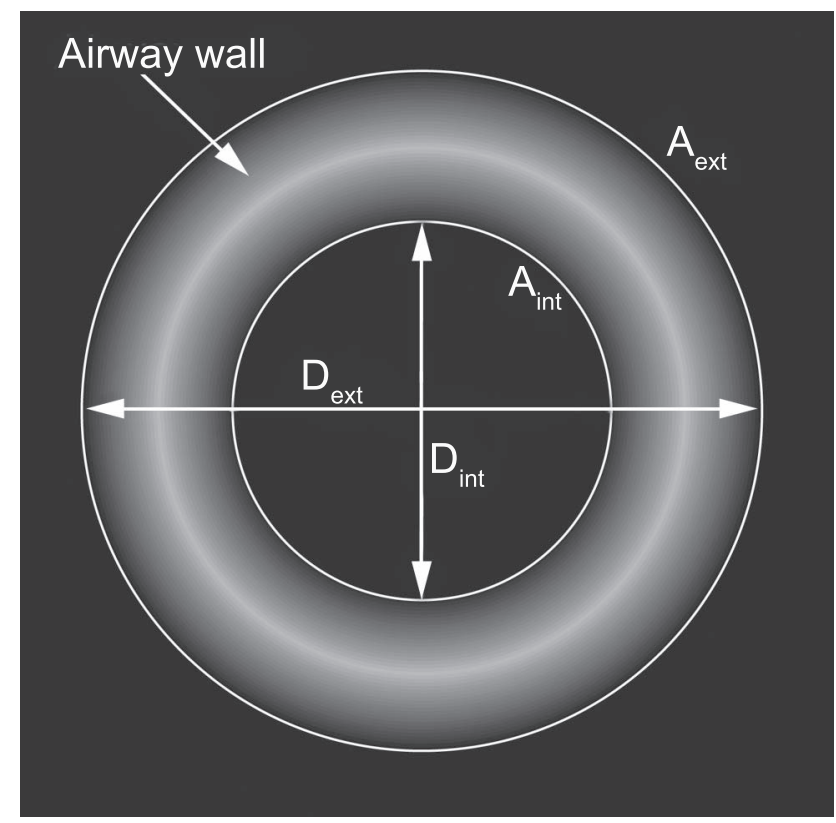

Fig. 5. Illustration of the airway wall thickness parameters. The wall thickness is computed as the difference between external diameter $\left(D_{\text {ext }}\right)$ and internal diameter $\left(D_{\text {int }}\right)$. $A_{\text {int }}=$ internal area, $A_{\text {ext }}=$ external area.

jority of subjects, this worked well; however, in some cases, it was necessary to manually edit the results of the lobe segmentation where the fissure was not well defined from the HRCT. In each case, the lobar segmentation results were visually assessed to ensure accuracy. 
Lung Sounds And Airway Morphology in COPD

Table 1. Demographic Data for the Sample Population

\begin{tabular}{|c|c|c|c|}
\hline Variables & $\begin{array}{l}\text { COPD } \\
(n=8)\end{array}$ & $\begin{array}{l}\text { Healthy } \\
\text { Smokers } \\
(n=9)\end{array}$ & $\begin{array}{c}\text { Healthy } \\
\text { Non-Smokers } \\
(n=9)\end{array}$ \\
\hline Females/males $(n)$ & $2 / 6$ & $7 / 2$ & $3 / 6$ \\
\hline $\operatorname{Age}^{*}(\mathrm{y})$ & $60(11)$ & $51(19)$ & $46(26)$ \\
\hline Weight* (kg) & $77.0(42.0)$ & $69.9(44.0)$ & $82.3(50.0)$ \\
\hline Height (m) & $1.69 \pm 0.08$ & $1.66 \pm 0.07$ & $1.72 \pm 0.08$ \\
\hline $\mathrm{BMI}^{*}\left(\mathrm{~kg} / \mathrm{m}^{2}\right)$ & $26.02(8.15)$ & $25.98(13.94)$ & $29.67(12.82)$ \\
\hline FVC (L) & $3.59 \pm 0.98$ & $3.89 \pm 0.85$ & $4.33 \pm 0.53$ \\
\hline $\mathrm{FEV}_{1}(\mathrm{~L})$ & $2.13 \pm 0.75 \dagger$ & $2.87 \pm 0.76$ & $3.54 \pm 0.49$ \\
\hline FVC (\% predicted) & $98.79 \pm 15.52 \neq$ & $117.93 \pm 13.64$ & $113.84 \pm 15.38$ \\
\hline $\mathrm{FEV}_{1}(\%$ predicted $)$ & $71.13 \pm 12.54 \S$ & $102.44 \pm 11.77$ & $107.90 \pm 11.09$ \\
\hline $\mathrm{FEV}_{1} / \mathrm{FVC}(\%)$ & $59.89 \pm 7.28 \S$ & $73.21 \pm 5.56 \dagger$ & $80.24 \pm 3.39$ \\
\hline $\begin{array}{l}\text { Peak expiratory } \\
\text { flow (\% predicted) }\end{array}$ & $68.24 \pm 13.63 \S$ & $107.18 \pm 18.06$ & $99.17 \pm 21.04$ \\
\hline \multicolumn{4}{|c|}{ 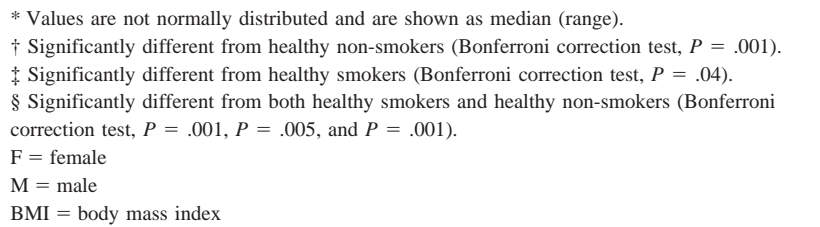 } \\
\hline
\end{tabular}

\section{Statistical Analysis}

Descriptive statistics are reported as mean \pm SD or median (range). One-way analysis of variance and post hoc comparison tests at the $95 \%$ significance level with a Bonferroni correction were used to compare the means of the 3 subject groups. The existence of a linear relationship between dependent variables (crackle $2 \mathrm{CD}$ or $\mathrm{NCpB}$ at each area over the chest wall) and independent variables (airway diameter, percentage wall area, and emphysema score at each corresponding auscultation area of the lung) was also tested by calculating Pearson correlation coefficient for the data from each generation of the airways in each lobe of the lung, down to generation 4 on the left and 5 on the right in 26 subjects.

\section{Results}

Demographic and pulmonary function test data are shown in Table 1. As expected, the COPD subjects presented with more impaired pulmonary function on average than both the healthy non-smokers and healthy smokers. Age did not have a normal distribution and is presented as median (range). There were no statistically significant differences in age across the groups $(P=.11)$ with mean rank age of 10.06 for healthy non-smoker, 13.11 for healthy smoker, and 17.81 for COPD, respectively. All results are presented as mean $\pm \mathrm{SD}$.
Table 2. Average Crackle Number per Breathing Cycle From the 6 Regions of the Chest Wall for Each Group

\begin{tabular}{lccc}
\hline \hline Area & $\begin{array}{c}\text { COPD } \\
(n=8)\end{array}$ & $\begin{array}{c}\text { Healthy Smokers } \\
(n=9)\end{array}$ & $\begin{array}{c}\text { Healthy Non-Smokers } \\
(n=9)\end{array}$ \\
\hline $\mathrm{AL}$ & $3.75 \pm 1.77$ & $3.52 \pm 1.28$ & $2.75 \pm 1.43$ \\
$\mathrm{LL}$ & $4.62 \pm 1.96$ & $4.25 \pm 1.27$ & $5.43 \pm 3.03$ \\
$\mathrm{PL}$ & $3.35 \pm 1.44$ & $4.78 \pm 2.22$ & $4.36 \pm 1.63$ \\
$\mathrm{AR}$ & $2.96 \pm 1.29$ & $3.71 \pm 1.68$ & $4.03 \pm 2.19$ \\
$\mathrm{LR}$ & $3.61 \pm 1.17$ & $5.09 \pm 1.48$ & $3.47 \pm 2.64$ \\
$\mathrm{PR}$ & $3.84 \pm 3.23$ & $4.54 \pm 1.70$ & $4.67 \pm 2.37$ \\
& & \\
$\mathrm{AL}=$ anterior left & & \\
$\mathrm{LL}=$ lateral left & & \\
$\mathrm{PL}=$ posterior left & & \\
$\mathrm{AR}=$ anterior right & & \\
$\mathrm{LR}=$ lateral right & & \\
$\mathrm{PR}=$ posterior right & & \\
\end{tabular}

Table 3. Average Crackle 2-Cycle Duration From the 6 Regions of the Chest Wall for Each Group

\begin{tabular}{lccc}
\hline \hline Area & $\begin{array}{c}\text { COPD } \\
(n=8)\end{array}$ & $\begin{array}{c}\text { Healthy Smokers } \\
(n=9)\end{array}$ & $\begin{array}{c}\text { Healthy Non-Smokers } \\
(n=9)\end{array}$ \\
\hline AL & $15.74 \pm 1.02$ & $15.63 \pm 0.83$ & $16.00 \pm 1.42$ \\
LL & $13.97 \pm 1.75^{*}$ & $15.64 \pm 1.31$ & $15.81 \pm 1.30$ \\
PL & $13.62 \pm 1.61$ & $14.89 \pm 1.48$ & $13.27 \pm 2.97$ \\
AR & $15.76 \pm 1.44$ & $16.39 \pm 0.95$ & $16.40 \pm 1.43$ \\
LR & $15.59 \pm 1.06$ & $14.51 \pm 1.00$ & $15.42 \pm 1.75$ \\
PR & $13.87 \pm 2.12$ & $15.00 \pm 1.02$ & $13.48 \pm 2.13$
\end{tabular}

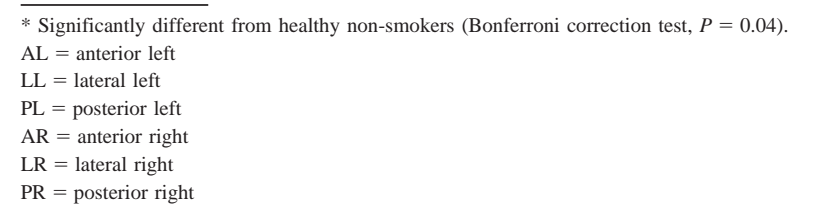

Crackle characteristics are presented in Tables 2 and 3. One-way analysis of variance revealed that the $\mathrm{NCpB}$ was not significantly different among the 3 groups at any region of the chest wall. Crackle $2 \mathrm{CD}$ was also not significantly different among the 3 groups in recordings from most regions of the chest wall (AL, AR, LR, PL, and PL). However, there was a significant difference in crackle $2 \mathrm{CD}$ at LL $(P=.03)$. Post hoc comparison across the 3 groups incorporating a Bonferroni correction showed that crackle $2 \mathrm{CD}$ in LL was marginally lower in COPD (13.97 \pm 1.75$)$ than in healthy non-smokers $(15.81 \pm 1.30, P=.04)$.

The average measures for airway diameter and percentage wall area are presented in Tables 4 and 5. One-way analysis of variance did reveal that there was a significant difference in airway diameter at generation 3 of right middle lobe (G3RML, $P=.03$ ). There were also some significant differences in percentage wall area at specific generations and lobes. These were: generation 3 of left upper lobe (G3LUL, $P=.02$ ); generation 4 of left lower lobe 
Table 6. Data for Emphysema Score in Each Lobe of the Lung

\begin{tabular}{lccc}
\hline \hline Area & $\begin{array}{c}\text { COPD } \\
(n=8)\end{array}$ & $\begin{array}{c}\text { Healthy Smokers } \\
(n=9)\end{array}$ & $\begin{array}{c}\text { Healthy Non-Smokers } \\
(n=9)\end{array}$ \\
\hline LUL & $2.16-25.55$ & $0.51-15.73$ & $0.61-4.88$ \\
LLL & $3.04-21.91$ & $0.14-7.77$ & $0.27-7.40$ \\
RUL & $0.38-48.71$ & $0.04-5.49$ & $0.38-3.78$ \\
RML & $1.05-21.91$ & $0.12-9.05$ & $2.21-11.32$ \\
RLL & $2.12-17.94$ & $0.06-7.56$ & $0.46-4.42$ \\
& & \\
Data are presented as percentage ranges. & \\
LUL $=$ left upper lobe & & \\
LLL $=$ left lower lobe & & \\
RUL $=$ right upper lobe & & \\
RML $=$ right middle lobe & & \\
RLL $=$ right lower lobe & & \\
\end{tabular}

Table 7. Pearson Correlation Coefficient (r) and $P$ Value for Significant Correlation Between Crackle Characteristics and Airway Measurements and Emphysema Score

\begin{tabular}{lrc}
\hline \hline \multicolumn{1}{c}{ Variables } & r & $P$ \\
\hline Crackle 2CD at AR and airway diameter at G2RUL & 0.44 & .03 \\
Crackle 2CD at AR and percentage wall area at right & -0.42 & .03 \\
main bronchus & & \\
Crackle 2CD at AR and percentage wall area at G2RUL & -0.40 & .04 \\
Crackle 2CD at AR and emphysema score at RUL & -0.47 & .01 \\
NCpB at AR and percentage wall areas at G2RUL & -0.49 & .01 \\
NCpB at LL and airway diameter at G4LLL & 0.48 & .01
\end{tabular}

Crackle $2 \mathrm{CD}=$ crackle 2-cycle deflection

$\mathrm{AR}=$ anterior right

$\mathrm{G} 2 \mathrm{RUL}=$ generation 2 right upper lobe

RUL $=$ right upper lobe

$\mathrm{NCpB}=$ mean number of crackles per breathing cycle

$\mathrm{G} 4 \mathrm{LLL}=$ generation 4 left lower lobe

\section{Multiple Correlation Tests}

There were 100 correlation tests performed at LUL and RLL among 5 variables; 2 with crackle $2 \mathrm{CD}$ and $\mathrm{NCpB}$ at each auscultation area and one with airway diameter, percentage wall area from each generation of LUL and RLL, and emphysema score at LUL and RLL. There were 81 and 84 correlation tests performed at LLL and LGL among 5 variables; 2 with crackle $2 \mathrm{CD}$ and $\mathrm{NCpB}$ at each auscultation area and one with airway diameter, percentage wall area from each generation of LLL and LGL, and emphysema score at LLL. There were 36 correlation tests performed at RML among 5 variables; 2 with crackle $2 \mathrm{CD}$ and $\mathrm{NCpB}$ at each auscultation area and one with airway diameter, percentage wall area from each generation of RML, and emphysema score at RML. There were 49 correlation tests performed at RUL among 5 variables; 2 with crackle $2 \mathrm{CD}$ and $\mathrm{NCpB}$ at each auscultation area and one with airway diameter, percentage wall area from each generation of RUL, and emphysema score at RUL. There were some significantly positive and negative correlations between airway measurements, emphysema score, and crackle characteristics, as shown in Table 7. These correlations were mostly observed in the RUL.

\section{Discussion}

This study was the first to investigate the relationship between airway geometry measured by HRCT and lung crackles measured by CALSA in vivo in human lungs. Previous animal research to study crackle mechanisms has been reported, but animal models may not mimic the human airway. ${ }^{7,8}$

The main results of the study are the lack of statistically significant differences in $\mathrm{NCpB}$ or crackle 2CD between the groups studied. There were some positive correlations noted between crackle characteristics and airway parameters. However, as this was the first study of this nature, multiple correlations were conducted to explore the potential relationships, with the inherent limitations of multiple testing; therefore, further research needs to be conducted to confirm these correlations.

The mechanism to generate crackles in COPD is uncertain, but can be explained by the following processes. Chronic inflammation and airway wall thickening decrease the lumen diameter of the airways, leading to an accumulation of secretions, ${ }^{20}$ and the formation of a fluid bridge within the airways. ${ }^{21}$ These processes can lead to closed airways by the fluid-film collapse mechanism. ${ }^{21}$ The destruction of lung parenchyma (emphysema) leads to the loss of structural support of the airway wall from pleural pressure, which also means that airways tend to close. ${ }^{21}$ During subsequent lung inflation, these closed airways are forced to break the fluid bridges to re-open, generating crackle sounds audible at the chest wall. However, this process requires sufficient air flow to break the fluid bridges, and, because COPD is associated with air flow limitation, the number of crackles that can be detected may be limited. This could provide some physiological underpinning of the negative correlation we found between $\mathrm{NCpB}$ and the percentage wall area at generation 2 of RUL, because thicker airway walls are less compliant and tend to pull the airway open, making it less likely to collapse (even if there is secretion blocking, or loss of support from parenchymal destruction). ${ }^{22}$ Because crackles are generated by the reopening of collapsed airways, fewer crackles would be generated under these conditions.

It was observed from the HRCT that there was a large degree of anatomical variation of the right upper lobe bronchus (G2RUL) of the tracheobronchial tree (Table 4 and Fig. 6), compared with the other branches of the airway that were considered. It has been reported that the generation of crackle sounds depends on the air flow; 3,7,8,23 therefore, because this airway is responsible for supplying 

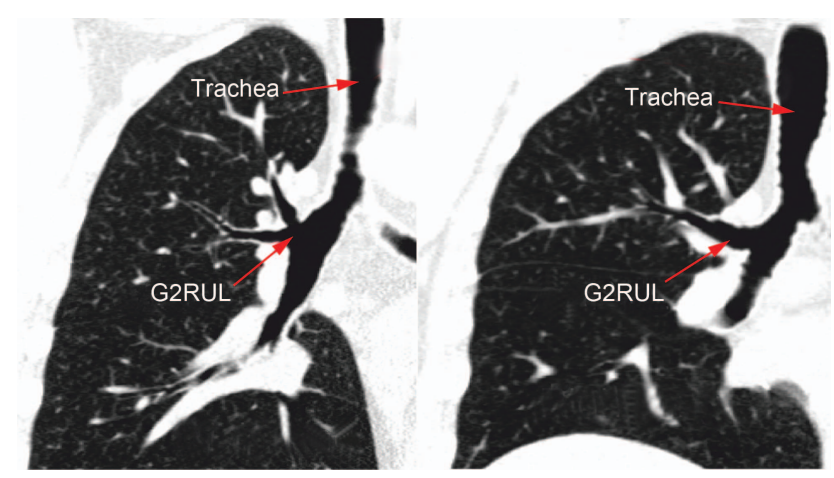

Fig. 6. Variation of RUL branch. Figure shows the very short RUL branch on the left compared with a clearly defined RUL branch on the right. G2RUL = generation 2 right upper lobe.

the air flow for the right upper lobe, it seems reasonable to assume that variations in the morphology could lead to significant changes in the air flow and hence the crackle characteristics.

If the relationships observed in this study are genuine, they suggest that thicker airway walls and higher emphysema scores are associated with crackles of shorter duration. These findings were in contrast with those reported by Jones and colleagues, ${ }^{23}$ who varied air flow at different levels into a simulated airway tube to generate crackles and reported that crackle generation depends on air flow, with low air flow generating crackles of longer duration. This disparity may be because our study was conducted in vivo, in the human lung, whereas the study by Jones and colleagues $^{23}$ used a simulated lung, which cannot provide the support of parenchymal tissues which affects the generation of lung sounds including crackles. However, there is no current theory to support our findings, so we cannot rule out the possibility that these correlations are occurring by chance.

A substantial limitation of this study was the number of multiple correlations that were tested, which meant that the possibility that any of the apparent correlations were occurring by chance could not be ruled out. There were 49 hypotheses to test the relationship between crackle characteristics at AR and airway measurements and emphysema score at RUL. When Bonferroni correction was applied to correct for the multiple correlations, with a significance of 0.05 , the null hypothesis could be rejected if the $P$ value was less than .001 . The $P$ values found in this study were in the range .01 to .04 and therefore too high to reject the null hypothesis. There was also a small sample size of only 26 subjects which resulted in low statistical power. The acceptable power in a clinical trial study is $80 \%$. To achieve this power with the correlation coefficient (r) of 0.4 (found in this study) a sample size of 47 subjects is needed as recommended by the Centre for Clinical Research and Biostatistics at the Chinese Univer-

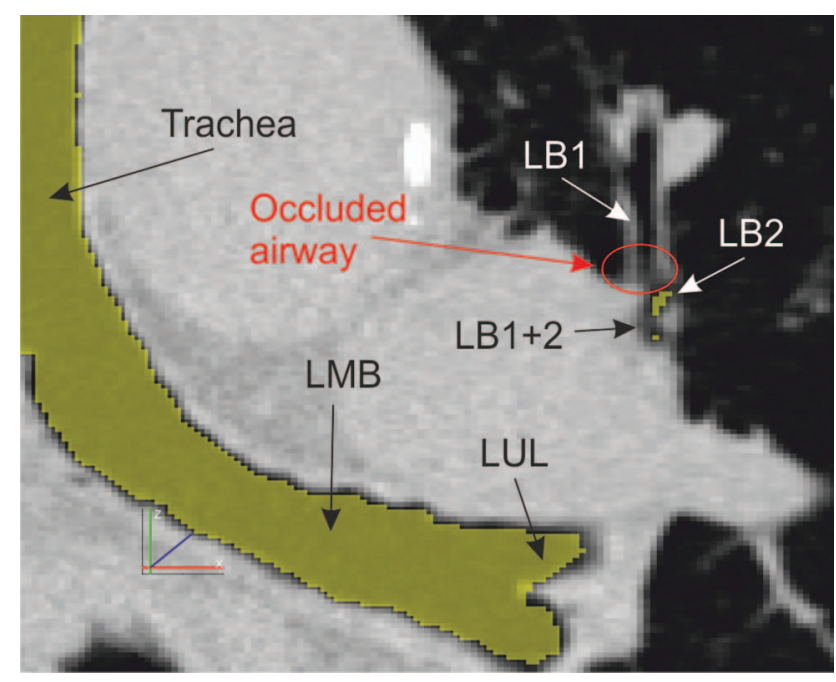

Fig. 7. High-resolution computed tomography image (coronal plane) showing detail of the left lung with an occluded airway (LB1). The occlusion (red oval) is indicated by the increased tissue density (brighter), compared with the airway lumen which appears very dark, or black. The airways that were detected by the Pulmonary Workstation 2 software have been highlighted in yellow. LMB = left main bronchus, LUL = left upper lobe, LB1 $+2=$ left bronchus 1 and 2, LB1 and LB2 = left segmental bronchi.

sity of Hong Kong (Hong Kong, People's Republic of China) (http://www.cct.cuhk.edu.hk/stat/other/correlation. htm, Accessed April 28, 2014). Thus, it is suggested that crackle characteristics could not be used to indicate COPD by statistical correlation. To avoid multiple correlation tests, we propose in the future that the correlation between crackle characteristics and airway morphology be investigated at only one specific generation, namely, that most affected in COPD (airway diameter less than $2 \mathrm{~mm}$ ).

There are also limitations within this research regarding the comparison of lung sounds with airway measurements. The HRCT procedure used in this study only provides data from the lungs, and hence measurements of the airways at suspended full inspiration. The lung sound recordings, however, were made during slightly deeper than normal breathing. This has resulted in a comparison of airway measurements from static snapshots of HRCT with lung sound data recorded during dynamic respiration. It is therefore not possible to use the HRCT to detect the exact cause of the crackle sounds detected, such as fluid bridges or collapsed airways, although such features are sometimes visible on the HRCT scan (see Fig. 7). Likewise, the use of a single-channel recording system, although selected to reflect common clinical practice in auscultation, means that the generation location within the lung of the crackle sounds detected cannot be ascertained and therefore cannot be related to specific features in the HRCT data.

The HRCT analysis by which the measures of airway wall geometry and emphysema score were derived was 
also not without limitations. The measurement of airway wall thickness, for example, was limited by the size of the voxels obtained from the HRCT reconstruction. In this study, the HRCT slice thickness was $0.7 \mathrm{~mm}$ and varied between approximately 0.6 and $0.8 \mathrm{~mm}$ in the transverse plane (as the reconstruction was fixed to a $512 \times 512$ voxel matrix, the size of the voxels had to change to accommodate patients of varying sizes). For the larger airways, the voxels will be sufficiently small that only a minimal bias will be introduced into the airway wall thickness measurement. However, as the airways become smaller and the wall thickness approaches the dimensions of a single voxel, the degree of measurement bias will increase. Furthermore, there is a substantial degree of anatomical variation between individuals in terms of the tracheobronchial tree, especially in terms of the dimensions of certain airways, such as the RUL branch. It is therefore not possible to attribute all of the variations in geometry between the healthy and COPD subject groups included in this study to the COPD disease process. Some will be natural variations between individuals.

The crackle analysis algorithm used in this study was developed following the study by Vannucini and colleagues, ${ }^{24}$ which followed the definition of crackles given by Murphy et al. ${ }^{5}$ Vannucini and colleagues ${ }^{24}$ reported a sensitivity of $84 \%$ and specificity of $89 \%$ to identify crackles. One weakness of this approach is the need to analyze the first derivative of the signal to identify potential crackles, as the first derivative of the signal is prone to significant sensitivity to contaminating noise. Furthermore, rulebased crackle identification processes are less reliable in the presence of multiple overlapping crackles, where characteristic features of one crackle may be masked by the sound of another. The algorithm applied in this study appeared biased toward the detection of coarse as opposed to fine crackles, perhaps because fine crackles often do not meet the requirement of a peak amplitude of twice the signal mean amplitude. This could explain why no fine crackles were observed in this study.

A further limitation of this study was the lack of age matching between the subject groups, which resulted in the COPD group being older than the healthy non-smoker group, although the difference was not statistically significant in this sample (possibly due to the small sample size). It has been reported that aging can cause alveolar enlargement, which could lead to the appearance of emphysema on HRCT. ${ }^{25}$ However, there have been no reports of age-related damage to the airway walls ${ }^{26}$ or crackle characteristics. It was therefore considered that the age difference probably had little impact on measurements related to airway wall geometry. No statistically significant difference was observed in the emphysema score or crackle characteristics between the groups; however, some statistically significant differences were found in airway wall thickness. The authors believe that the age difference was therefore unlikely to have had any significant impact on the findings of this study. However, further studies should be conducted with subject age matching between the groups to minimize any potential impact.

Finally, there were a small number of subjects in each group for this study, leading to reduced statistical power to detect any differences between the subject groups. Future studies will involve larger subject numbers to test the relationships between lung sounds and airway morphology.

\section{Conclusions}

This is the first study to report on the relationship between lung sounds and the underlying geometry of the airways in vivo of human lung. The results from this study suggest no significant relationship between crackle characteristics quantified using computer-aided lung sound analysis, and airway variables and emphysema score measured by HRCT. It is uncertain whether no such relationship exists or whether our small sample was unrepresentative of the wider COPD population. Therefore, this study provides no conclusive evidence that crackle characteristics are related to HRCT variables in COPD.

\section{ACKNOWLEDGMENTS}

The authors acknowledge the assistance of Peter Howarth MBBS DM FRCP.

\section{REFERENCES}

1. Murphy RL. In defense of the stethoscope. Respir Care 2008;53(3): 355-369.

2. Piirilä $P$, Sovijärvi AR, Kaisla T, Rajala HM, Katila T. Crackles in patients with fibrosing alveolitis, bronchiectasis, COPD, and heart failure. Chest 1991;99(5):1076-1083.

3. Piirilä $P$, Sovijärvi AR. Crackles: recording, analysis and clinical significance. Eur Respir J 1995;8(12):2139-2148.

4. Pasterkamp H, Kraman SS, Wodicka GR. Respiratory sounds: advances beyond the stethoscope. Am J Respir Crit Care Med 1997; 156(3):974-987.

5. Murphy RL Jr, Del Bono EA, Davidson F. Validation of an automatic crackle (rale) counter. Am Rev Respir Dis 1989;140(4):10171020.

6. Marques A, Bruton A, Barney A. The reliability of lung crackle characteristics in cystic fibrosis and bronchiectasis patients in a clinical setting. Physiol Meas 2009;30(9):903-912.

7. Alencar AM, Buldyrev SV, Majumdar A, Stanley HE, Suki B. Avalanche dynamics of crackle sound in the lung. Phys Rev Lett 2001; 87(8):88101-88104.

8. Alencar AM, Buldyrev SV, Majumdar A, Stanley HE, Suki B. Perimeter growth of a branched structure: application to crackle sounds in the lung. Phys Rev E 2003;68(1):0011909/1-12.

9. Turato G, Zuin R, Saetta M. Pathogenesis and pathology of COPD. Respiration 2001;68(2):117-128.

10. Sauret V, Halson PM, Brown IW, Fleming JS, Bailey AG. Study of the three-dimensional geometry of the central conducting airways in man using computed tomographic (CT) images. J Anat 2002;200(Pt 2):123-134. 


\section{Lung Sounds AND Airway Morphology IN COPD}

11. Tiddens HA, Paré PD, Hogg JC. Hop WC, Lambert R, De Jongste JC. Cartilaginous airway dimensions and airflow obstruction in human lungs Am J Respir Crit Care Med 1995;152(1):260-266.

12. Han MK, Bartholmai B, Liu LX, Murray S, Curtis JL, Sciurba FC, et al. Clinical significance of radiologic characterizations in COPD. COPD 2009;6(6):459-467.

13. de Jong PA, Müller NL, Paré PD, Coxson HO. Computed tomography imaging of the airways: relationship to structure and function. Eur Respir J 2005;26(1):140-152.

14. Hasegawa M, Nasuhara Y, Onodera Y, Makita H, Nagai K, Fuke S, et al. Airflow limitation and airway dimensions in chronic obstructive pulmonary disease. Am J Respir Crit Care Med 2006;173(12): 1309-1315.

15. Matsuoka S, Kurihara Y, Nakajima Y, Niimi H, Ashida H, Kaneoya $\mathrm{K}$. Serial change in airway lumen and wall thickness at thin-section CT in asymptomatic subjects. Radiology 2005;234(2):595-603.

16. Kitaguchi Y, Fujimoto K, Kubo K, Honda T. Characteristics of COPD phenotypes classified according to the findings of HRCT. Respir Med 2006;100(10):1742-1752.

17. Coxson H. Lung parenchyma density and airwall thickness in airway diseases. Breathe 2012;9(1):36-45.

18. Marques A, Bruton A, Barney A, Hall A. Are crackles an appropriate outcome measure for airway clearance therapy? Respir Care 2012; 57(9):1468-1475
19. Coxson HO. Quantitative computed tomography assessment of airway wall dimensions: current status and potential applications for phenotyping chronic obstructive pulmonary disease. Proc Am Thorac Soc 2008;5(9):940-945.

20. Hogg JC, Chu F, Utokaparch S, Woods R, Elliott WM, Buzatu L, et al. The nature of small-airway obstruction in chronic obstructive pulmonary disease. N Engl J Med 2004;350(26):2645-2653.

21. Heil M, Hazel AL, Smith JA. The mechanics of airway closure. Respir Physiol Neurobiol 2008;63(1-3):214-221.

22. Diaz AA, Come CE, Ross JC, San José Estépar R, Han MK, Loring $\mathrm{SH}$, et al. Association between airway caliber changes with lung inflation and emphysema assessed by volumetric $\mathrm{CT}$ in subjects with COPD. Chest 2012;141(3):736-744.

23. Jones AY, Jones RD, Kwong K, Burns Y. The effect on sound generation of varying both gas flow rate and the viscosity of sputumlike gel in a simple tubular model. Lung 2000;178(1):31-40.

24. Vannuccini L, Rossi M, Pasquali G. A new method to detect crackles in respiratory sounds. Technol Health Care 1998;6(1):75-79.

25. Janssens JP, Pache JC, Nicod LP. Physiological changes in respiratory function associated with ageing. Eur Respir J 1999;13(1):197-205.

26. Fukuchi $Y$. The aging lung and chronic obstructive pulmonary disease: Similarity and difference. Proc Am Thorac Soc 2009;6(7):570572. 